\title{
Cool Stars in the EUV: Spectral and Structural Variability
}

\author{
ALEXANDER BROWN \\ Center for Astrophysics and Space Astronomy, \\ University of Colorado, Campus Box 389, Boulder, CO 80309-0389, USA
}

\begin{abstract}
EUV observations of RS CVn binaries and M dwarf flare stars are used to illustrate the variability of coronal emission seen in EUVE spectra and photometry. The EUVE emission line spectra show that the quiescent coronae of active stars are extremely hot ( $\geq 10$ million $\mathrm{K}$ ) and flares, which are even hotter, are common. Spectral sequences showing the evolution of flare temperature and density are presented. Very high coronal electron densities $\left(\log N_{e} \sim 13\right)$ have been detected during $M$ dwarf flares. EUVE data for the RS CVn binary HR1099 are compared with those from simultaneous observations in other spectral regions, including $\mathrm{X}$-ray $(A S C A)$, ultraviolet (IUE), and radio (VLA, AT) data. These combined data show that the peak coronal temperature is above the sensitivity of $E U V E$ and that the time of maximum flare brightness is spectral region dependent.
\end{abstract}

\section{Introduction}

The study of stellar coronae has been hindered greatly by a lack of detailed information on the physical properties, such as the ranges of temperature and density, present in the outer atmospheres of stars other than the Sun. This situation has changed radically with the availability of coronal EUV emission line spectra from the Extreme Ultraviolet Explorer (EUVE) satellite (Bowyer \& Malina 1991). Earlier X-ray telescopes, such as ROSAT and Einstein, provided data of such low spectral resolution that, while the coronal fluxes could be estimated, attempts to characterise coronal temperature distributions were crude if not altogether misleading. Pointed $E U V E$ observations consist of data from four coaligned instruments; three spectrometers and the Deep Survey (D/S) photometer. These spectrometers provide coverage over the following wavelength ranges: $70-190 \AA$ ("short wavelength," SW); 140-380 $\AA$ ("medium wavelength," MW); and 280-760 ("long wavelength," LW). The Deep Survey photometer observes through a broadband Lexan/B filter with a passband almost identical to the SW spectrometer and can thus provide higher time resolution information on coronal flux variations than possible from the measurements of individual spectral features.

The spectral region observable with EUVE (70-700 $\AA$ ) contains many important emission lines formed at temperatures between a few $10^{4}$ and a few $10^{7} \mathrm{~K}$. Thus stellar EUV spectra are very sensitive probes of the dominant conditions within stellar outer atmospheres (See e.g. Brown 1994). However, much of this spectral interval is difficult to observe due to interstellar absorption. Consequently, for all but a few of the nearest stars, very few emission lines are seen above $400 \AA$. For solar-like stars EUV emission is dominated by plasma at temperatures of a few $10^{6} \mathrm{~K}$ and strong emission lines of ions such as Fe IX-XII are observed. Very active stars, including the RS CVn binaries and flare stars discussed in this paper, show strikingly different spectra dominated by lines of Fe XVIII-XXIV formed at temperatures near or above $10^{7} \mathrm{~K}$, as shown in Figure 1 . 


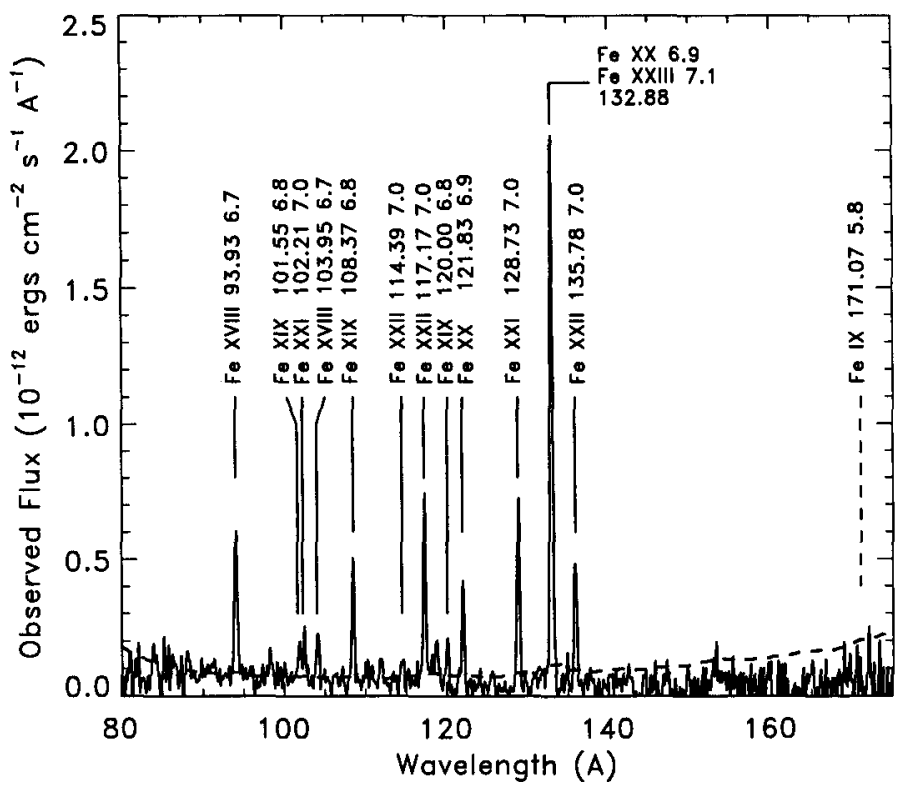

Figure 1. The integrated EUVE short wavelength spectrum of $\sigma^{2} \mathrm{CrB}$ obtained on 1994 February 16-21 during a $144 \mathrm{ks}$ exposure. The dashed line shows the $3 \sigma$ detection threshold. Prominent emission line are identified by ion, wavelength, and temperature $(\log T)$ of formation. The expected position of the strongest Fe IX line is noted.

\section{Coronal Variability of RS CVn Binaries}

RS CVn binaries are close binaries with typical orbital periods of a few days. Due to tidal action that acts to enforce orbital and rotational synchronism many stars in these systems rotate extremely rapidly and thus show very high levels of coronal activity.

As an illustration of RS CVn EUV coronal variability I present results from an observation of $\sigma^{2}$ CrB obtained over a 4.5 day period (Brown, Linsky, \& Dempsey 1994). The stars in this system have spectral types of $\mathrm{F} 6 \mathrm{~V}$ and a $\mathrm{G} 0 \mathrm{~V}$, and an orbital period of 1.14 days $(=98.5 \mathrm{ks})$. Being a dwarf system the rotational periods of the stars are $2 \%-3 \%$ longer than the orbital period. $\sigma^{2} \mathrm{CrB}$ is at a distance of $21 \mathrm{pc}$ and thus has a low interstellar hydrogen column density, allowing good transmission of EUV photons. The $E U V E$ exposure times were $\sim 140 \mathrm{ks}$ and spanned over 4 binary orbital periods; therefore, flaring and rotation modulation are easily distinguished. That direct analysis of the integrated mean EUVE emission line spectra (illustrated in Fig. 1) would be injudicious can be seen from the D/S light curve presented in Figure 2. Two intervals of flaring occur separated by two binary orbital periods, with peak increases of factors of 5 and 3 relative to quiescence. In both cases the outbursts last $\sim 11 \mathrm{hrs}$ and show evidence for the occurrence of multiple flares. Figure 3 shows the spectra from the two flare events and the three surrounding intervals of quiescent emission. Note that the emission line fluxes increase significantly during the flare events, showing that these are not artifacts within the $\mathrm{D} / \mathrm{S}$ data. However, while the five spectra vary in strength, there is no dramatic change in the appearance of the spectra between flares and quiescence. This behaviour is typical of RS CVn flares and indicates that the quiescent temperature is close to or above the maximum temperature sensitivity of EUVE spectra. EUVE MW 


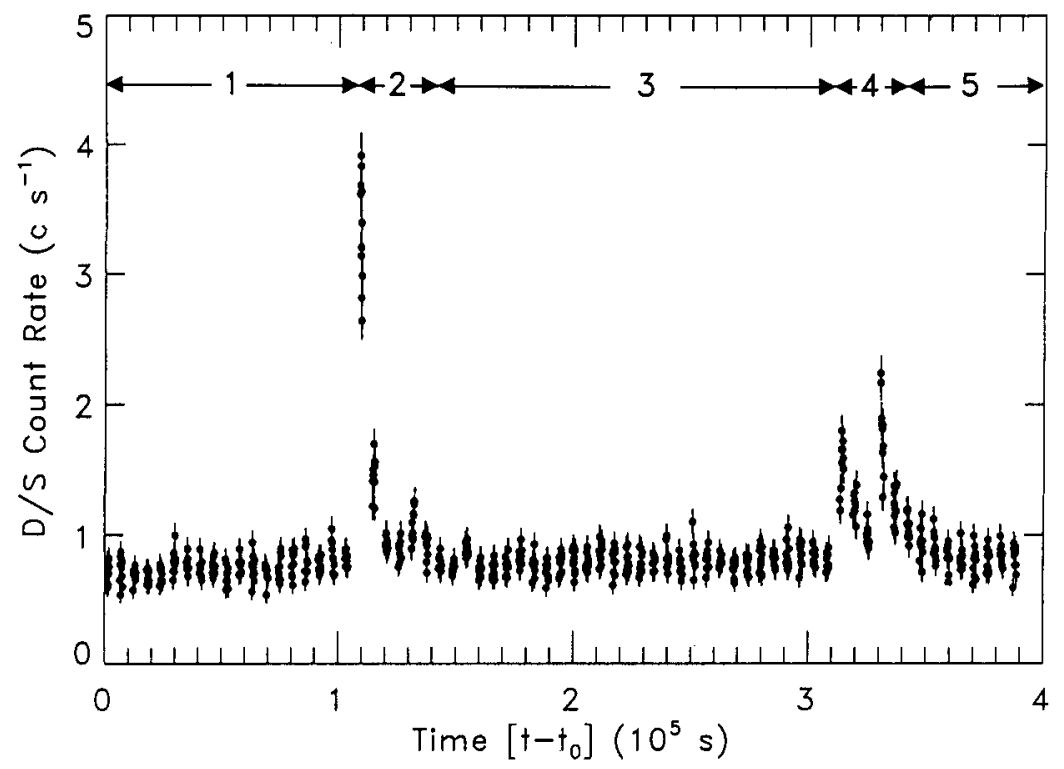

Figure 2. The EUVE Deep Survey light curve of $\sigma^{2} \mathrm{CrB}$ obtained over the period 1994 February 16-21. The five time intervals used for temporal analysis are shown at the top.

spectra are dominated by He II $304 \AA$ but also contain the hottest lines visible in $E U V E$ spectra, i.e., Fe XXIV (192 $\AA$ and $255 \AA$ ) formed at $\log T_{e}=7.2$ or higher. The MW spectra of most stars show a similar behaviour during flares with fluxes rising but no appearance of hotter lines. However, the MW spectra of $\sigma^{2} \mathrm{CrB}$ are unique to this point in showing dramatic (a factor of 10 for the first flare) increases in Fe XXIV line fluxes during the two flare events. This implies that the quiescent coronal temperature of $\sigma^{2}$ CrB peaks at about $\log T_{e}=7.0$, and higher temperatures present in flare plasma are seen as large increases in Fe XXIV emission.

Large EUV flares are common in the coronae of RS CVn binaries. During EUVE observations of HR1099 (G5 IV + K1 IV, orbital period 2.84 days) obtained over 9 days in 1993 September and 1994 August at least five flare outbursts occurred, with four long outbursts lasting between 12 and 24 hours. In contrast a 3 day observation in 1992 October showed only weak flaring with one definite hour-long flare occurring at phase $\phi=0.1$. Drake et al. (1994) found possible evidence for rotational modulation due to a long-lived coronal structure by comparing both EUVE pointed and all-sky survey data taken approximately 2 months apart. However, flare-like brightenings could not be discounted as the cause, reinforcing the need for long, continuous observations in variability analyses.

In all three years the onset of flares occur in two narrow phase intervals, $\phi=0.1-0.3$ and $0.75-0.85$. It is as yet unclear whether this correlation is significant or how flaring might be related to orbital quadratures in HR1099. During this same time interval the location of starspots and active regions on the surface of the $\mathrm{K} 1$ star derived from optical data have changed significantly. 


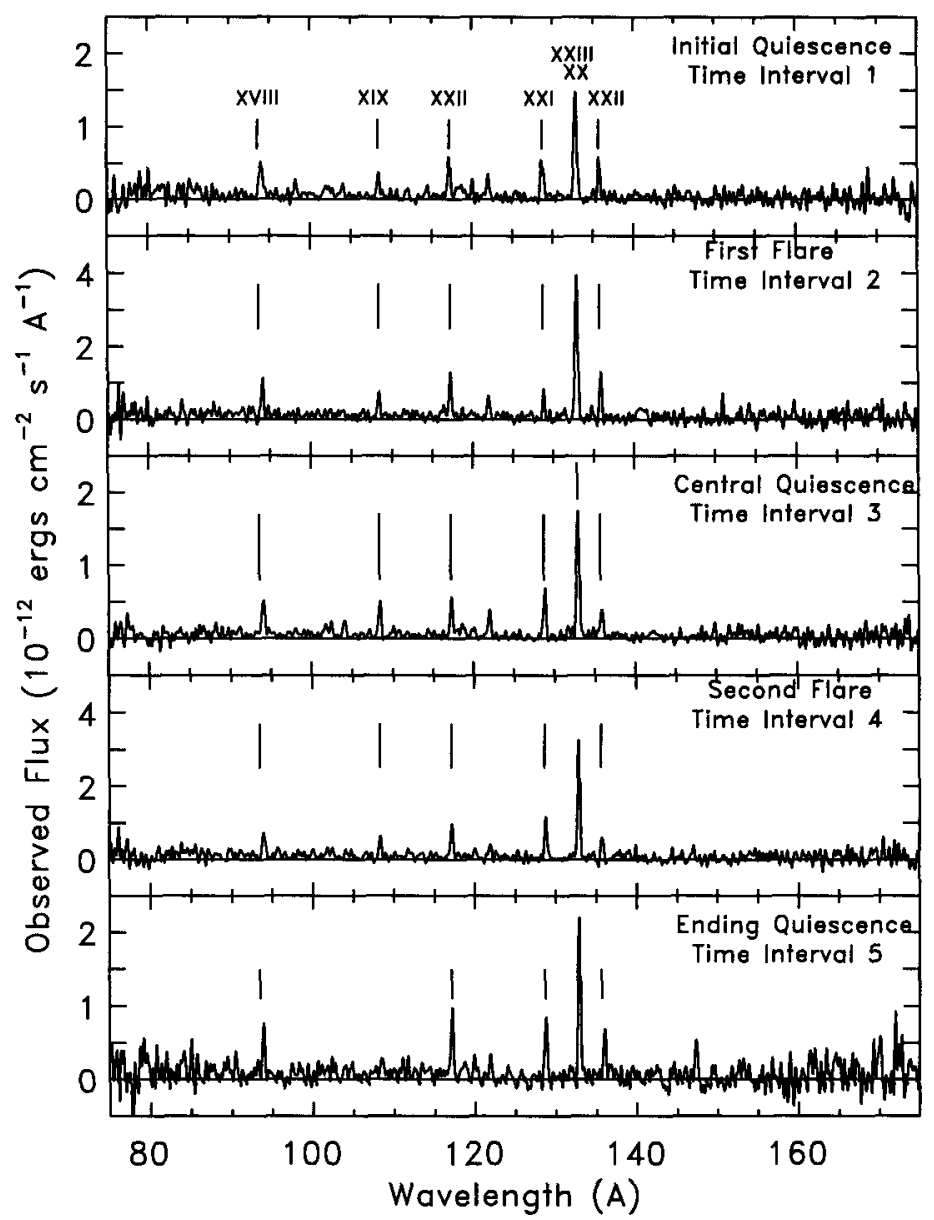

Figure 3. Time-resolved EUVE SW spectra of $\sigma^{2} \mathrm{CrB}$ showing the response of coronal emission lines due to stellar flares. Roman numerals indentify the ionisation stage of different Fe emission lines.

\section{EUV Flaring on dMe Flare Stars}

Flares on $\mathrm{M}$ dwarf stars have been known for almost 50 years due to the detection of optical $\mathrm{U}$ and $\mathrm{B}$ band enhancements associated with the impulsive phase of flares that last typically a few minutes. Similarly X-ray observations have shown numerous flares with typical timescales of minutes to hrs (see e.g. Pallavicini, Tagliaferri, \& Stella 1990). Consequently $E U V E$ observations of dMe stars were expected to provide significant new information on the coronal conditions during flares. This has proved correct with the detection of numerous EUV flares, e.g. flares on AU Mic (Cully et al. 1993), AD Leo (Hawley et al. 1995), EQ Peg (Monsignori-Fossi et al. 1995), EV Lac (Ambruster et al. 1994), and AT Mic (Monsignori-Fossi et al. 1994). Typically these flares have durations of hrs to more than a day. In Figure 4 the D/S light curve of a relatively short flare on EV Lac is shown. This flare has a simpler form than most EUV flares with a smooth 


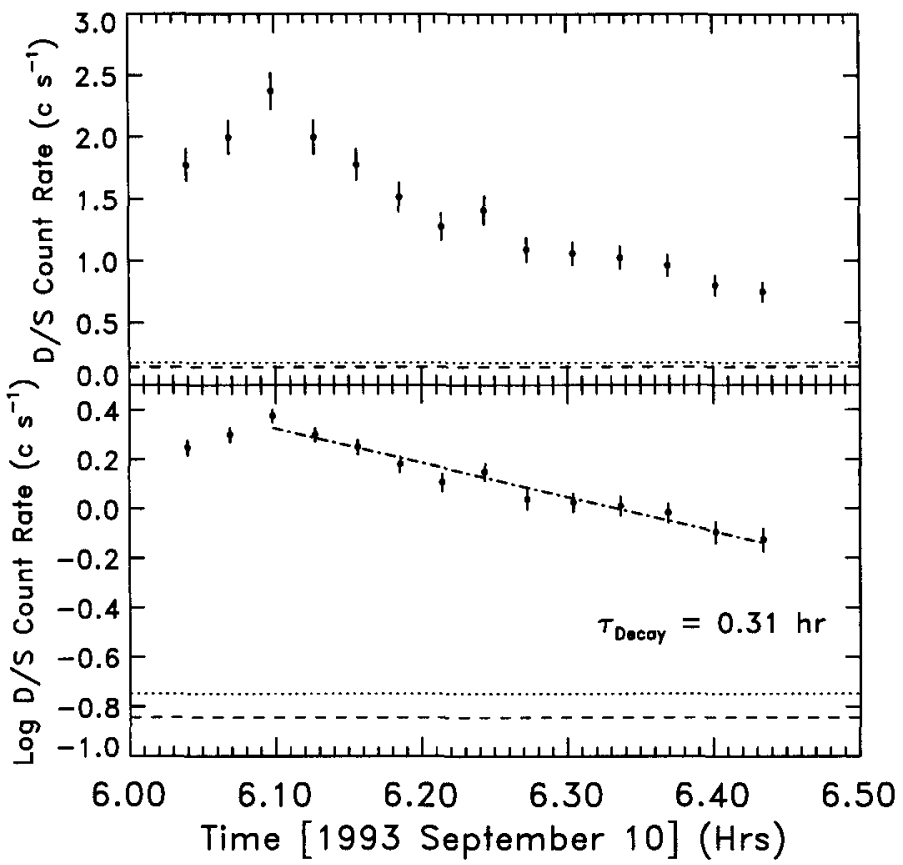

FIGURe 4. EUVE Deep Survey light curve of a flare on the M dwarf EV Lac. The dot-dashed line shows an exponential decay fit to the flare decay. The dotted line shows the mean D/S count rate for the complete observation, while the dashed line shows the mean count rate excluding this large flare.

decline that is almost exponential in form, with only slight evidence for additional flaring. However, many of the long duration flares, such as those of AU Mic and AD Leo, show the presence of strong flares superimposed on the decay of a larger flare. From the photometry alone it is not possible to determine the interrelationship between such flares. In the case of the $1992 \mathrm{July} 15 \mathrm{AU}$ Mic flare it is possible to determine the coronal ( $10^{7} \mathrm{~K}$ ) electron density during the course of the flare. Figure 5 shows the variation of the Fe XXII $114 \AA$ to $117 \AA$ ratio during different time intervals of the D/S variations (see Fig. 1 of Cully et al. 1993). High electron densities are found during the initial decay of the large flare and in the second shorter flare. These high densities indicate the presence of compact sources of intense magnetic heating. Interestingly, the peak of the large flare does not show high density, contrary to the results of Monsignori-Fossi \& Landini (1994). Such large densities during the flare decay suggest that continued heating of the plasma must be occurring contrary to the coronal mass ejection model of Cully et al. (1994).

\section{The Importance of Multiwavelength Observations for Studying Flare Phenomena}

While $E U V E$ has provided extremely important opportunities for the detailed study of stellar coronae over long time intervals, $E U V E$ spectra and photometry can still only provide a limited insight into the physical processes operating in these magnetically controlled and highly dynamic plasmas. Observations involving data from a range of 


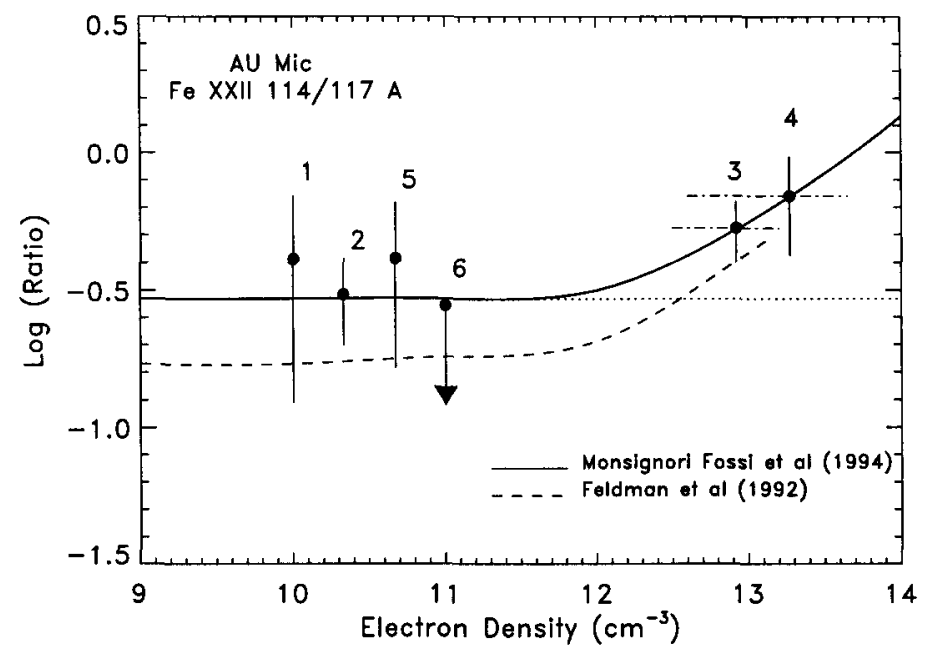

FIGURE 5. Estimates of the log electron density in the corona of AU Mic based on the ratio of Fe XXII 114.4 $\AA$ to $117.2 \AA$ for different intervals in the $1992 E U V E$ observation. The solid and dashed curves show two calibrations of this ratio; the Monsignori-Fossi et al. calibration is much more consistent with the data.

spectral regions can lead to an even more detailed investigation of coronal phenomena. Routinely we try to obtain observations simultaneous with $E U V E$ in spectral regions such as the radio, ultraviolet, and X-ray to the extent that observatory schedules allow. As an example I show a portion of our 1994 August observations of HR1099 in Figure 6. This time interval covers a single day from a 5 day observation. The unique aspect of this time interval is the first simultaneous observation of a stellar corona by $A S C A$ and $E U V E$. $A S C A$ observes in the soft $\mathrm{X}$-ray region $(0.5-10 \mathrm{KeV})$. Figure 6 shows the presence of a small flare with a duration of $\sim 4 \mathrm{hrs}$ and a peak luminosity in the 1-300 $\AA$ region of $210^{31} \mathrm{ergs} \mathrm{s}^{-1}$. Analysis of the $A S C A$ spectra show that the hottest coronal component ranges from a quiescent temperature of $\log T_{e}=7.4$ to 7.5 at the peak of the flare, confirming that the peak temperature in many RS CVn flares is beyond the temperature sensitivity of $E U V E$. The relative timing of the flare in different spectral regions is particularly interesting. The flare timing and shape is almost identical in the $E U V E \mathrm{D} / \mathrm{S}$ and $A S C A$ SIS data, and this implies that both instruments are seeing the flare decay rather than the initial impulsive phase of the flare. The UV flare response is seen at the very onset of the flare rise in X-rays/EUV; although the 8 hour break in IUE coverage eliminates UV coverage of most of the flare. The $3 \mathrm{~cm}$ radio continuum emission, due to gyrosynchrotron emission from nonthermal coronal electrons, shows a flare starting roughly an hour earlier than in the other spectral regions.

\section{Summary}

- Most active cool stars (RS CVn binaries and flare stars) observed by EUVE have shown variability, usually in the form of readily identifiable stellar flares.

- RS CVn EUV flares typically have longer time scales than those on the Sun. EUV flares on dMe stars can also last up to a day but are typically somewhat shorter than RS CVn flares. 


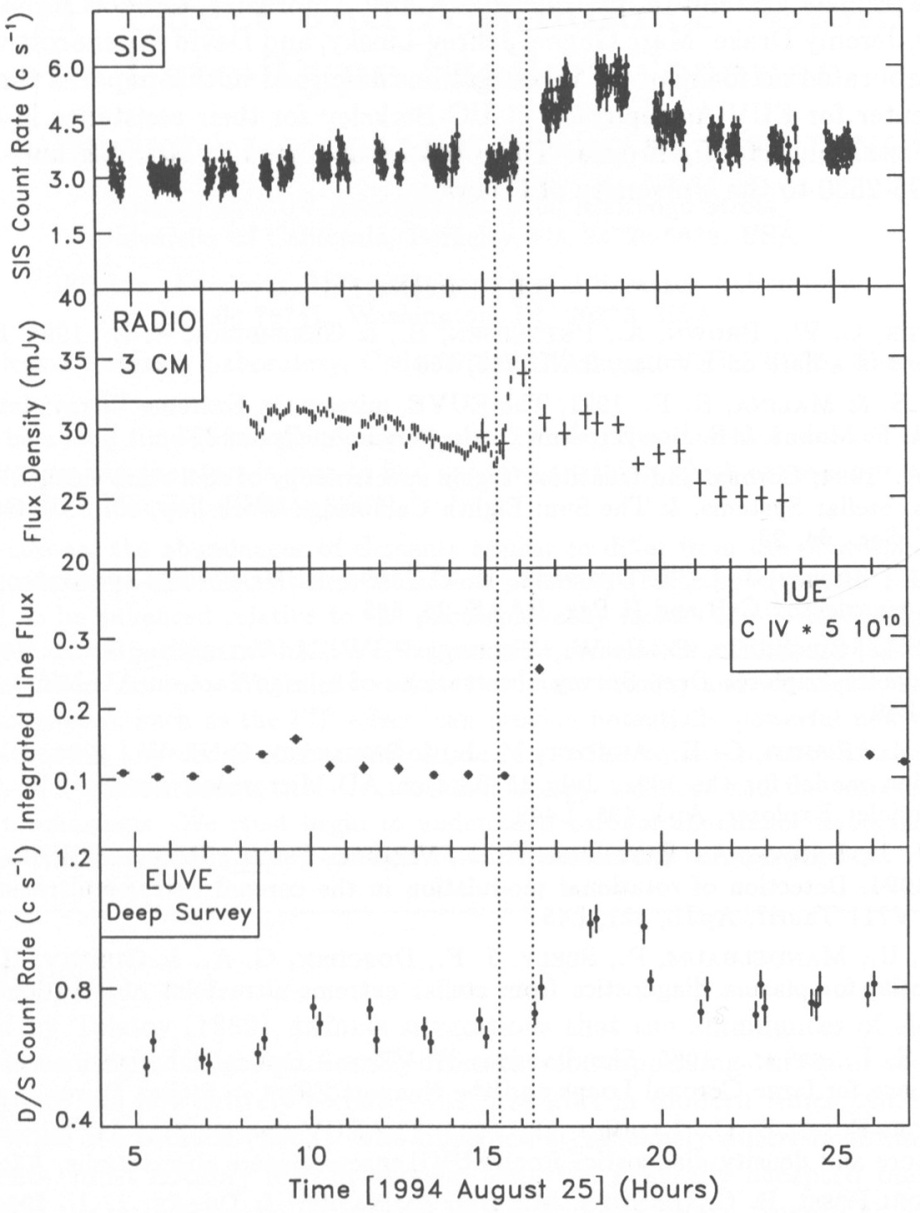

FIGURE 6. Simultaneous multispectral-region observations of the RS CVn binary HR1099 obtained on 1994 August 24. The data shown (from top to bottom) are soft X-ray count rate from the $A S C A$ SIS detector, $3 \mathrm{~cm}$ radio continuum flux density from the VLA (vertical lines) and AT (crosses), ultraviolet CIV line flux (formed at $10^{5} \mathrm{~K}$ ) from IUE SWP-LO spectra, and the EUVE D/S count rate. Dotted lines mark times of flare onset.

- RS CVn flares seem to occur preferentially at particular orbital phases, although this result is based on as yet limited statistics.

- Coronal temperatures of active stars are close to the upper limit of the $E U V E$ temperature sensitivity; usually flares increase the flux in all emission lines but no new hotter lines appear.

- $E U V E$ does not see the flare impulsive phase.

- High ( $\left.\log N_{e} \sim 13\right)$ electron densities have been seen during short rapid flares. Rapid spiky" flares are often seen superimposed on the decays of large outbursts.

- $E U V E$ is an excellent instrument for studying coronal flares, particularly due to the long (days) time intervals that can be studied. 
I am extremely grateful to my colleagues Carol Ambruster, Grodon Bromage, Robert Dempsey, Jeremy Drake, Marc Gagne, Jeffrey Linsky, and David Wonnacott with whom I have collaborated on many of the investigations described in this paper. I thank the staff of the Center for EUV Astrophysics at UC Berkeley for their assistance in the analysis and understanding of $E U V E$ data. This work is supported by NASA grants NAG5-2259 and NAG5-2530 to the University of Colorado.

\section{REFERENCES}

Ambruster, C. W., Brown, A., Pettersen, B., \& Gershberg, R. E. 1994, EUVE observations of a flare on EV Lac, BAAS, 26, 866

BowYer, S. \& MalinA, R. F. 1991, The EUVE, mission, in Extreme Ultraviolet Astronomy, ed. R. F. Malina \& S. Bowyer, New York: Pergamon Press, 397

BRown, A. 1994, Coronal and transition region spectroscopy of cool stars using EUVE, in Cool Stars, Stellar Systems, \& The Sun: Eighth Cambridge Workshop, ed. J.-P. Caillault, ASP Conf. Ser., 64, 23

Brown, A., Linsky, J. L., \& DemPSEY, R. C. 1994, EUVE coronal spectroscopy of the RS $\mathrm{CVn}$ binaries $\sigma^{2} \mathrm{CrB}$ and II Peg, BAAS, 26, 865

Cully, S. L., Siegmund, O. H. W., Vedder, P. W., \& Vallerga, J. V. 1993, Extreme Ultraviolet Explorer Deep Survey observations of a large flare on AU Microscopii, ApJL, 414, L49

Cully, S. L., Fisher, G. H., Abbott, M. J., \& Siegmund, O. H. W. 1994, A coronal mass ejection model for the 1992, July 15 flare on AU Microscopii observed by the Extreme Ultraviolet Explorer, ApJ, 435, L449

Drake, J. J., Brown, A., Patterer, R. J., Vedder, P. W., Bowyer, S., \& Guinan, E. F. 1994, Detection of rotational modulation in the coronal extreme-ultraviolet emission from V711 Tauri?, ApJL, 421, L43

Feldman, U., Mandelbaum, P., Seely, J. F., Doschek, G. A., \& Gursky, H. 1992, The potential for plasma diagnostics from stellar extreme-ultraviolet observations, ApJS, 81, 387

HAWLEY, S. L., ET AL. 1995, Simultaneous EUVE and Optical Observations of AD Leonis: Evidence for large Coronal Loops and the Neupert Effect in Stellar Flares, ApJ, in press

Monsignori-Fossi, B. C., LANDINI, M. 1994, The EUV Spectrum of AU Microscopii: Temperature and density diagnostics from EUVE spectrometers observations, A\&A, 284, 900

Monsignori-Fossi, B. C., Landini, M., Del Zanna, G., \& Drake, J. J. 1994, The EUV Spectrum of AT Mic. in Cool Stars, Stellar Systems, \& The Sun: Eighth Cambridge Workshop, ed. J.-P. Caillault,. ASP Conf. Ser., 64, 44

Monsignori-Fossi, B. C., Landini, M., Fruscione, A., \& Dupuis, J. 1995, Extreme Ultraviolet Spectroscopy and Photometry of EQ Pegasi, A\&A, in press

Pallavicini, R., Tagliaferri, G., \& Stella, L. 1990, X-ray emission from solar neighbourhood flare stars: A comprehensive survey of EXOSAT results, A\&A, 228, 403 